\title{
La ecología de los medios: metadisciplina compleja y sistémica
}

Octavio Islas ${ }^{1}$

Recibido: 2015-08-29

Enviado a pares: 2015-08-29
Aprobado por pares: 2015-09-19

Aceptado: 2015-09-21

DOI: 10.5294/pacla.2015.18.4.5

Para citar este artículo / To reference this article / Para citar este artigo Islas, 0. (Diciembre de 2015). La ecología de los medios: metadisciplina compleja y sistémica. Palabra Clave, 18(4), 1057-1083. DOI: 10.5294/pacla.2015.18.4.5

\section{Resumen}

La ecología de los medios no es una corriente teórica de las ciencias de la comunicación ni se limita y agota en la obra y pensamiento de Marshall McLuhan. La ecología de los medios es una metadisciplina compleja y sistémica, cuyo objeto de estudio son los cambios que han producido las tecnologías y los medios de comunicación en las sociedades a lo largo de la historia. Este artículo problematiza los alcances de la ecología de medios en tanto corriente teórica y forma epistemológica y hace una apuesta que trasciende la visión tradicional e involucra un amplio número de ciencias, como la gramática, la retórica, la semiótica, la teoría de sistemas, la historia, la filosofía, la cibernética, las ciencias de la comunicación, las artes - la literatura - y, por supuesto, la tecnología misma.

\section{Palabras clave}

Ecología de los medios, metadisciplina, complejidad, Marshall McLuhan (Fuente: Tesauro de la Unesco).

1 Universidad de los Hemisferios, Ecuador. octavio.islas@itesm.mx 


\section{The Media Ecology: Complex and Systemic Meta-discipline}

\section{Abstract}

The media ecology is not a theoretical stream of communication sciences nor is it limited and exhausted by the work and thinking of Marshall McLuhan. The media ecology is a complex and systemic meta-discipline, whose object of study are the changes that technologies and media in societies have produced throughout history. In this paper we discuss the scope of media ecology as current theoretical and epistemological way and makes a bet that transcends the traditional view and involves a wide range of sciences such as grammar, rhetoric, semiotics, theory of systems, history, philosophy, cybernetics, communication sciences, arts and literature-and of course, the technology itself.

\section{Keywords}

Media ecology, meta-discipline, complexity, Marshall McLuhan (Source: Unesco Thesaurus). 


\section{A ecologia das mídias: metadisciplina complexa e sistêmica}

\section{Resumo}

A ecologia das mídias não é uma corrente teórica das ciências da comunicação nem se limita e se esgota na obra e no pensamento de Marshall McLuhan. A ecologia das mídias é uma metadisciplina complexa e sistêmica, cujo objeto de estudo são as mudanças que as tecnologias e os meios de comunicação têm produzido nas sociedades ao longo da história. Este artigo problematiza os avanços da ecologia das mídias enquanto corrente teórica e forma epistemológica, e faz uma aposta que transcende a visão tradicional e envolve um amplo número de ciências, como a gramática, a retórica, a semiótica, a teoria de sistemas, a história, a filosofia, a cibernética, as ciências da comunicação, as artes —a literatura—e, obviamente, a tecnologia em si.

\section{Palavras-chave}

Ecologia das mídias, metadisciplina, complexidade, Marshall McLuhan (Fonte: Tesauro da Unesco). 


\section{Introducción}

En la primera parte de este artículo, explico qué es la ecología de los medios (media ecology), refiero cuál es su objeto de estudio y concluyo que de ninguna manera admite ser considerada como una teoría más en las ciencias de la comunicación. La ecología de los medios debe ser reconocida como una metadisciplina compleja y sistémica que trasciende a las ciencias de la comunicación y al "mcluhanismo". En el segundo apartado, para afirmar a la ecología de los medios como metadisciplina compleja y sistémica, recupero su particular interpretación del tiempo histórico, destacando la importancia que admiten los ritmos informacionales en cada edad histórica. En el tercer apartado, explico la presencia del pensamiento complejo a lo largo de la historia y destaco que algunos rasgos del pensamiento complejo ya estaban presentes en culturas milenarias. A partir del siglo XVI, la ciencia empezó a nutrirse de relatos disciplinarios. La departamentalización de las ciencias relegó al pensamiento complejo. Sin embargo, el formidable desarrollo tecnológico alcanzado en décadas recientes propició las condiciones necesarias para el retorno del pensamiento sistémico y complejo. La cibernética (Wiener, 1981) legitimó el estatuto científico del pensamiento complejo. Niklas Luhmann extendió la teoría general de sistemas a la comprensión de los fenómenos sociales. Lance Strate, uno de los medioecologistas más importantes en la actualidad, ha dado cuenta de los estrechos vínculos que admiten determinadas tesis de McLuhan y Luhmann, así como profundas relaciones de conocimiento que es posible advertir entre la ecología de los medios, la teoría general de sistemas y la semántica general. Por último, en el libro Las leyes de los medios: la nueva ciencia, Marshall y Eric McLuhan introducen su tétrada, razonamiento definitivamente sistémico y complejo, destinada a explicar los efectos que producen los cambios tecnológicos en las sociedades a lo largo de la historia.

\section{Qué es la ecología de los medios}

La ecología de los medios también es conocida como Escuela de Toronto, Escuela de Nueva York, Escuela de San Luis, Escuela Norteamericana de Estudios Culturales y Mediología. Sin embargo, en cada una de las referidas "versiones" es posible advertir ciertos matices y, en algunos casos, abiertas diferencias en el significado y alcance de determinadas tesis y conceptos; 
las remediaciones, por ejemplo. En el sitio web de la Media Ecology Association (MEA) (http://www.media-ecology.org/), asociación fundada en 2000 por Neil Postman (1931-2003) y Lance Strate (1956), en la Universidad de Fordham (Nueva York), es posible consultar la definición ofrecida por Neil Postman (1931-2003), destacado educador y sociólogo estadounidense, profesor de la Universidad de Nueva York:

La ecología de los medios analiza cómo los medios de comunicación afectan la opinión humana, la comprensión, la sensación y el valor, y cómo nuestra interacción con los medios facilita o impide nuestras posibilidades de supervivencia. La palabra ecología implica el estudio de ambientes: su estructura, contenido e impacto en la gente. Un ambiente es un complejo sistema de mensajes que impone en el ser humano formas de pensar, sentir, y actuar. Estructura lo que podemos ver y decir y, por consiguiente, hacer. Asigna roles e incide en el ejercicio de estos. Especifica qué podemos y qué no podemos hacer. En algunas ocasiones, como es el caso de un tribunal, un salón de clases 0 una oficina, las especificaciones son formales y explícitas. En el caso de los ambientes mediáticos (libros, radio, cine, televisión, etcétera), las especificaciones, por lo general, son implícitas e informales, parcialmente ocultas, por el supuesto de que no estamos tratando con un ambiente, sino simplemente con una máquina. La ecología de los medios pretende hacer explícitas estas especificaciones, tratando de encontrar qué roles nos obligan a desempeñar los medios, cómo los medios estructuran lo que estamos viendo, y la razón por la cual estos nos hacen sentir y actuar de la manera en que lo hacemos. La ecología de los medios es el estudio de los medios como ambientes (Postman, s. f.). ${ }^{2}$

Robert K. Logan (1939), doctor en Física egresado del prestigioso Instituto Tecnológico de Massachusetts (MIT), discípulo de Marshall McLuhan y miembro de la segunda generación de la Escuela de Toronto ${ }^{3}-a$ pesar de su origen neoyorquino-, en uno de sus libros más interesantes, Understanding new media: extending Marshall McLuhan, presentó la siguiente definición de ecología de los medios:

2 El 16 de junio de 2000, Neil Postman dictó la conferencia inaugural que dio vida a la MEA, en Fordham, universidad ubicada en el Bronx, en Nueva York. El título de la conferencia magistral de Postman fue "The humanism of media ecology" (http://www.media-ecology.org/publications/MEA_proceedings/v1/humanism_of_media_ecology.html). Carlos Scolari incluyó esa conferencia en su libro sobre ecología de los medios.

3 Entre los principales miembros de la primera generación de la llamada Escuela de Toronto destacan: Harold Innis, Eric A. Havelock y Ted Carpenter. En la segunda generación, participaron Quentin Fiore, Kathy Hutchon, Eric McLuhan, Barrington Nevitt, Harley Parker, Bruce Powers y Wilfred Watson, y Bob Logan. 
Tradicionalmente, un sistema ecológico o ecosistema se refiere a un sistema biológico que consiste en un medio ambiente natural físico y los organismos vivos que habitan en ese entorno físico, así como las interacciones de todos los componentes del sistema. Un ecosistema de medios se define en analogía con un ecosistema biológico tradicional como un sistema formado por los seres humanos y los medios de comunicación y la tecnología a través de cual interactúan y se comunican entre sí. También incluye las lenguas con las que expresar y codificar la comunicación [...] Lengua y tecnologías de mediar y crear ambientes como los medios de comunicación. Medios y lenguajes son ambas técnicas y herramientas, como cualquier otra forma de tecnología. Medios de comunicación y las tecnologías son lenguajes de expresión, que al igual que una información se comunican con lenguaje de su semántica y la sintaxis propia y única. Dadas estas coincidencias, podemos afirmar que el estudio ecológico de los medios de comunicación no se puede restringir a los medios de comunicación en sentido estricto, sino que también debe incluir la tecnología y el lenguaje y las interacciones de estos tres ámbitos, que juntas forman un ecosistema de medios (Logan, 2010, pp. 33-34).

Fernando Gutiérrez (1972), mexicano, reconocido de los nuevos medios digitales y miembro del board directivo de la MEA, es autor de la siguiente definición:

La Ecología de los Medios es una metadisciplina que se encarga del estudio de un conjunto complejo de relaciones o interrelaciones entre símbolos, los medios y la cultura. La palabra ecología implica el estudio de los ambientes y sus interrelaciones: contenido, estructura, e impacto social. Un ambiente mediático es aquel que deriva de las interrelaciones entre el hombre y las distintas tecnologías de comunicación como: libros, radio televisión, internet. La ecología mediática se refiere al estudio de las técnicas, modos de información y códigos de comunicación como parte principal de un ambiente interrelacionado que proyecta diferentes efectos en un contexto determinado (citado en Islas, 2014). ${ }^{4}$

Wikipedia (s. f. a) atribuye a Lance Strate, profesor de la Universidad de Fordham, destacado discípulo de Neil Postman, primer presidente de la MEA, la siguiente definición, que en 2015 Carlos Scolari recuperó en su libro Ecología de los medios, cuyo punto de partida fue el texto

4 Fernando Gutiérrez, funcionario del Tecnológico de Monterrey, campus Santa Fe, es miembro directivo del board de la MEA. En la décimo sexta convención de la MEA, fue distinguido con el reconocimiento Louis Forsdale. 


\title{
"Ecología de los medios: mapa de un nicho teórico", publicado por los Quaderns del CAC.
}

\begin{abstract}
La ecología de los medios es el estudio de los entornos mediáticos, la idea de que la tecnología y las técnicas, los modos de información y los códigos de comunicación juegan un rol fundamental en los asuntos humanos. La ecología de los medios es la Escuela de Toronto y la Escuela de Nueva York. Es determinismo tecnológico, hard y soft, y evolución tecnológica. Es lógica de los medios, teoría del medium, mediología. Son los estudios de McLuhan, las investigaciones sobre oralidad y alfabetismo, los estudios culturales americanos. Es gramática y retórica, semiótica y teoría de los sistemas, historia y filosofía de la tecnología. Es lo postindustrial y lo posmoderno, lo prealfabético y prehistórico (Strate, Understanding MEA, 1999, citado en Scolari, 2010, p. 22).
\end{abstract}

Por otro lado, Wikipedia ofrece una interesante descripción de la ecología de los medios y su objeto de estudio, explicando, además, la forma como fue gestado el término ecología de los medios y cómo esta pretende explicar los cambios sociales que han derivado de las tecnologías a lo largo de la historia:

De acuerdo con la Media Ecology Association, el término ecología de los medios se puede definir como "el estudio de los entornos de los medios de comunicación, la idea de que la tecnología y las técnicas, los modos de información y los códigos de comunicación desempeñan un papel de liderazgo en los asuntos humanos". La teoría de la ecología de los medios está centrada en los principios de que la tecnología no solo influye profundamente a la sociedad, sino que también controla prácticamente todos los ámbitos de la vida. Se trata de un estudio de cómo los medios de comunicación y los procesos de comunicación afectan la percepción y la comprensión humana. El término primero fue introducido formalmente por Neil Postman en 1968, mientras que la teoría fue propuesta por Marshall McLuhan en 1964. Para fortalecer esta teoría, McLuhan y Quentin Fiore afirman que los medios de comunicación han definido la esencia de la sociedad con el reconocimiento de cuatro épocas: la era tribal, la era del alfabeto, la era de la imprenta y la era electrónica, que corresponden al modo dominante de comunicación de sus respectivos tiempos. McLuhan sostiene que los medios de comunicación actúan como extensiones de los sentidos humanos en cada época, y las tecnologías de la comunicación son la causa principal del cambio social. Para entender cómo los medios de comunicación producen grandes cambios estructurales 
en la perspectiva humana, McLuhan clasifica los medios como calientes o fríos. Por medios calientes se refiere a una comunicación de alta definición que exige poca participación de la audiencia, mientras que los medios de comunicación fríos exigen la participación activa de la audiencia. McLuhan, con su hijo Eric McLuhan, amplió la teoría en 1988, desarrollando una manera de profundizar en el reconocimiento de los efectos que produce la tecnología en la sociedad. La tétrada, como un concepto organizado que permite sustentar y reconocer, con las leyes de los medios de comunicación, el pasado, el presente y los futuros efectos de los medios de comunicación. La ecología de los medios es un término controvertido en los estudios de los medios de comunicación, ya que tiene diferentes significados en contextos europeos y norteamericanos. En América del Norte, se refiere a un campo interdisciplinario de la teoría de los medios de comunicación, que implica el estudio de los entornos, mientras que la versión europea de la ecología de los medios comprende una investigación materialista de los sistemas de medios de comunicación como sistemas dinámicos y complejos (Wikipedia, s. f. b).

La ecología de los medios efectivamente se cuestiona por los efectos que se desprenden del desarrollo tecnológico a lo largo de la historia. El término ecología de los medios fue introducido por Neil Postman en una conferencia que dictó en un acto organizado por la National Council of Teachers of English, en 1968. Como atinadamente destaca Carlos Scolari:

El propio Postman reconocía que Marshall McLuhan lo había utilizado a principios de esa década, en la época de mayor brillo intelectual del canadiense (The Gutenberg Galaxy es de 1962 y Understanding Media de 1964) [...] durante su conferencia, Postman definió a la ecología de los medios como "el estudio de los medios como ambientes" ("the study of media as environments") (Scolari, 2010, p. 18).

En 1971 Neil Postman fundó el programa Ecología de los Medios en la Universidad de Nueva York. Sin embargo, a Marshall McLuhan correspondió el mérito de haber definido con anterioridad el objeto de estudio de la ecología de los medios. Las tecnologías - y debemos tener presente que los medios de comunicación son tecnologías - afectan la percepción y la comprensión humana, como destacó Marshall McLuhan en 1964, en la primera edición de su cuarto libro Understanding media: the extension of man: "Los efectos de la tecnología no se producen al nivel de las opiniones o de los conceptos, sino que modifican los índices sensoriales, o pautas de 
percepción, regularmente y sin encontrar resistencia” (McLuhan, 1996, p. 39). Sobre la importancia de Marshall McLuhan en la gestación y desarrollo de la ecología de los medios, Lance Strate afirmó:

Ningún individuo es más central en la ecología de los medios que
McLuhan, no porque él fuera el primero en emplear este punto de
vista, sino más bien porque él lo popularizó, y produjo la primera
gran síntesis del pensamiento ecológico en medios. Para algunos, el
mcluhanismo o los estudios de McLuhan son suficientes en sí mis-
mos, y todas las respuestas se pueden encontrar en sus escritos.
Para otros, eran las preguntas que se formuló y que tenían el ver-
dadero significado, ya que abrió un campo relativamente nuevo de
estudio, sondeado territorios desconocidos, generando entusiasmo,
y sirvió como fuente de inspiración. Para la gran mayoría, fue este
libro, publicado por primera vez en 1964, lo que los convirtió en el
estudio de los entornos multimedia (Wikipedia, s. f. b).

La ecología de los medios estudia el impacto que producen los medios - y las tecnologías en general — sobre la cultura de las sociedades a lo largo de la historia. Las tecnologías producen ambientes mediáticos que eluden la fácil percepción de las personas. La ecología de los medios no es una escuela o corriente teórica de las ciencias de la comunicación, como suponen algunos académicos e investigadores de esta disciplina en Iberoamérica, particularmente quienes en años recientes se han interesado por la obra de Marshall McLuhan (1911-1980) y por la ecología de los medios. Además la ecología de los medios no se agota en el pensamiento de Herbert Marshall McLuhan (1911-1980), quien insistentemente ha sido señalado por no pocos académicos e investigadores de las ciencias de la comunicación en Iberoamérica como uno de los pensadores emblemáticos del llamado "determinismo tecnológico" y el "tecnooptimismo".

La ecología de los medios no necesita profundizar en la "metáfora ecológica” para tomar la forma de un conjunto científico más ordenado y completo. Y no necesita hacerlo porque la complejidad inherente a su objeto de estudio trasciende a las ciencias de la comunicación y al "mcluhanismo". Como atinadamente fue asentado en la anterior cita tomada de Wikepedia, en la tradición norteamericana la ecología de los medios comprende un enfoque interdisciplinario, que le permite analizar los ambientes mediáticos. La tradición 
europea instala a la ecología de los medios en la perspectiva misma de los sistemas complejos.

\section{La interpretación del tiempo histórico}

Para afirmar a la ecología de los medios como metadisciplina compleja y sistémica, en primer lugar, resulta indispensable destacar su particular interpretación del devenir histórico. Las teorías no disponen ni ofrecen una interpretación estructurada del tiempo histórico. Su alcance explicativo es limitado y reposan sobre las interpretaciones ofrecidas por grandes paradigmas, como el materialismo histórico, en el cual el concepto 'modo de producción' permite explicar el devenir histórico. La historia de la humanidad hasta nuestros días —-sentenció Marx en El manifiesto comunistaes la historia de las luchas de clases. Los modos de producción expresan la interrelación entre las fuerzas productivas y las relaciones sociales de producción. Desde tal perspectiva, a lo largo de la historia, es posible identificar los siguientes modos de producción: comunismo primitivo o modo de producción asiático — donde no existió lucha de clases-, esclavismo, feudalismo, capitalismo - y de acuerdo con Lenin el imperialismo, la fase superior del capitalismo - y el comunismo. El socialismo representa la primera fase del comunismo. La dictadura del proletariado es la segunda etapa, y finalmente se consuma la plena abolición de la lucha de clases en la tercera etapa, el comunismo.

Marshall McLuhan propuso una interpretación distinta para comprender el devenir histórico, a partir del reconocimiento de distintas edades comunicativas — referidas estas en la última cita tomada de Wikipedia-: una edad tribal, la edad del alfabeto, la edad de la imprenta — era mecánica o Galaxia Gutenberg - y la edad eléctrica. En el libro Understanding New Media: Extending Marshall McLuhan (2010), Robert Logan se atribuyó el mérito de haber añadido dos edades comunicativas, las cuales, según Logan, no fueron consideradas por Marshall McLuhan: la era preverbal o mimética y la era digital. Sin embargo, al contrario de lo que sostiene Logan, Marshall McLuhan sí consideró una era tribal, equivalente a la era preverbal o mimética que refiere Logan. Además, en el libro Understanding Media, McLuhan anticipó el advenimiento de una edad "poseléctrica", a la cual designó con el término information age (edad de la información). 
McLuhan no solo propuso las referidas etapas históricas, además estableció que a cada etapa histórica corresponde un determinado "ritmo informacional” y un medio de comunicación principal. En la primera edad —la edad tribal—, la circulación de la información era muy lenta. Tomó miles de años poder inventar el primer alfabeto fonético. En tal periodo histórico, el equilibrio sensitivo fue absoluto. Con la invención del alfabeto fonético se aceleró la circulación de la información, y el sentido del oído empezó a ser relegado a un segundo plano por el sentido de la vista. En la Edad Media, el desarrollo de las comunicaciones y los sistemas de transporte incrementaron la velocidad en la circulación de la información. La imprenta fue el medio de comunicación dominante en la era mecánica o Galaxia Gutenberg. El sentido de la vista relegó aún más al sentido del oído. En la era mecánica fue posible acceder a la secuencialidad. La división del trabajo y el maquinismo fueron lógico resultado del nuevo orden impuesto. En la edad eléctrica — que dio inicio con la invención del telégrafo-, la información circuló aún más rápido, lo cual implicó mayor complejidad. Posiblemente la televisión admitiría ser considerada como el principal medio de comunicación en la edad eléctrica. En el libro Understanding Media: The Extension of Man, McLuhan afirmó que la televisión era extensión del sentido del tacto, pues este involucra todos los sentidos.

El concepto 'edad de la información' fue recuperado por Daniel Bell (1919-2011) en el libro El advenimiento de la sociedad posindustrial (1973). A Bell es atribuido el concepto 'sociedad de la información'. También Alvin Toffler, en el libro La tercera ola (1979), especuló sobre una edad de la información. Vale la pena destacar que en ese libro Toffler introdujo el concepto de 'prosumismo' y anticipó el advenimiento de los prosumidores. En la edad de la información, la información circula aún más rápido, y en buena medida ello ha sido posible gracias a internet, medio que ha propiciado la instantaneidad informativa absoluta. Los teléfonos inteligentes han extendido a internet, el cual se ha desplazado, con la inteligencia artificial, a dispositivos diversos; internet de los objetos. Sobre la importancia de los ritmos informacionales y la complejidad de la organización social resultante, McLuhan afirmó:

El aumento de velocidad desde lo mecánico a la forma eléctrica instantánea invierte la explosión en implosión. En la actual edad 
eléctrica, las energías en implosión o contracción. De nuestro mundo chocan con los antiguos patrones de organización, expansionistas y tradicionales [...] De hecho, lo que genera nuestra preocupación por la población no es el aumento de las cantidades, sino el hecho de que todo el mundo ha de vivir en la más estrecha proximidad creada por nuestra implicación eléctrica y recíproca en la vida de los demás (McLuhan, 1996, p. 55).

En el materialismo histórico, el fin de la historia fue decretado en el siglo XIX por Carlos Marx y Federico Engels, quienes anticiparon el advemiento del comunismo y el fin de la lucha de clases en las sociedades. En la ecología de los medios, el futuro de ninguna manera se encuentra resuelto. Al contrario, el futuro permanece abierto a los efectos que producirán nuevas tecnologías en la cultura de nuevas sociedades, definitivamente mucho más complejas.

\section{La ecología de los medios y el pensamiento complejo y sistémico}

El pensamiento sistémico representa uno de los grandes paradigmas del conocimiento a lo largo de la historia. Es posible advertir la presencia de algunos de sus principales rasgos en civilizaciones muy antiguas, las cuales reconocieron un mundo holístico en la naturaleza y emprendieron la búsqueda de regularidades en los fenómenos, a partir de observar las relaciones entre los elementos que intervenían en su ocurrencia, aventurándose a predecir el desarrollo de fenómenos futuros a partir del reconocimiento de las regularidades identificadas (considérese en McLuhan el reconocimiento de patrones).

En el siglo XVI, el conocimiento sistémico empezó a ser desplazado por paradigmas unidisciplinarios que partieron de visiones del mundo que suponen la búsqueda de explicaciones mucho más sencillas, estables y objetivas. En la producción de relatos especializados - que con el tiempo facilitaron la actual fragmentación científica y la consecuente especialización departamental - destacan los siguientes autores: Francis Bacon (1561-1626), con el Novum organon — publicado en 1620—; René Descartes (1596-1650), con el Discurso del método — publicado en 1637-; 
Thomas Hobbes (1588-1679), con El Leviatán —publicado en 1651-, e Isaac Newton (1642-1727), con Philosophiae naturalis principia mathematica, publicado en 1687.

Sin embargo, el formidable desarrollo tecnológico alcanzado durante las recientes décadas favoreció el retorno del pensamiento sistémico y complejo. La cibernética reinstaló al pensamiento complejo en el imaginario científico. Esta fue concebida por Norbert Wiener (1864-1964), destacado matemático estadounidense, quien laboró en el programa U.S. National Defense Research Comittee con Vannevar Bush (1890-1974). Bush fue el principal responsable del mencionado programa y también es recordado por la iniciativa Memex, concepto precursor de la World Wide Web (WWW). Wiener, quien distinguía dos grandes etapas en el desarrollo de la civilización: la edad mecánica y la edad eléctrica, diferenciadas por el tipo de instrumentos empleados por el hombre —notable similitud con McLuhan—, introdujo el concepto 'cibernética' en la primera edición del libro Cibernética o el control y la comunicación en animales y máquinas:

Hasta hace muy poco tiempo no existía una voz que comprendiera ese conjunto de ideas; para poder expresarlo todo mediante una palabra, me vi obligado a inventarla. De ahí: cibernética, que derivé de la voz griega kubernetes o timonel, la misma raíz de la cual los pueblos de Occidente han formado gobierno y sus derivados (Wiener, 1981, p. 17).

Sobre Wiener y el origen de la cibernética, el belga Armand Mattelart —uno de los principales críticos de Marshall McLuhan- destacó:

\begin{abstract}
En 1948, Norbert Wiener (1894-1964) publica Cibernética o control y comunicación en animales y máquinas. Esta obra, en la que se entrecruzan observación de procesos de control fisiológicos y neurofisiológicos (contracción del músculo cardiaco, prestaciones del sistema nervioso como un todo integrado) y formalización de una teoría general sobre los sistemas tecnológicos de control, es el punto de partida de la ciencia del pilotaje o cibernética (2002, p. 59).
\end{abstract}

Wiener introdujo los conceptos de feedback, o retroacción y 'cantidad de información’ en la aún incipiente teoría de la comunicación. A Wiener, además, le debemos una primera distinción entre máquinas analógicas y digitales. En el libro Cibernética y sociedad, Wiener definió el concepto 'información', incorporando en su definición el término complejidad: 
Damos el nombre de información al contenido de lo que es objeto de intercambio con el mundo externo, mientras nos ajustamos a él y hacemos que se acomode a nosotros. El proceso de recibir y utilizar informaciones consiste en ajustarnos a las contingencias de nuestro medio y de vivir de forma efectiva dentro de él. Las necesidades y la complejidad de la vida moderna plantean a este fenómeno del intercambio de informaciones demandas más intensas que en cualquier otra época; la prensa, los museos, los laboratorios científicos, las universidades, las bibliotecas y los libros de texto han de satisfacerlas o fracasarán en sus propósitos. Vivir de manera efectiva significa poseer la información adecuada. Así, pues, la comunicación y la regulación constituyen la vida interior del hombre, como de su vida social (1981, p. 19).

Las notables contribuciones de Wiener fueron recuperadas ya avanzada la década de 1940, por dos destacados matemáticos estadounidenses: Claude Elwood Shannon (1916-2001) — considerado "padre de la teoría de la información”, quien en 1948 publicó en dos partes el artículo "Una teoría matemática de la comunicación” en el Bell System Technichal Journal-, y Warren Weaver (1894-1978). A partir de las propuestas formuladas por Shannon y Weaver, derivaron interesantes modelos de comunicación, que incluyeron conceptos, como información, fuente, mensaje, transmisor, señal, canal, ruido, receptor, destino, probabilidad de error, codificar, decodificar, ruta de la información y capacidad del canal. Shannon y Weaver también fueron blanco de los cuestionamientos de algunos académicos e investigadores de las ciencias de la comunicación en Iberoamérica, quienes les estigmatizaron como autores de la teoría matemática de la información y precursores del paradigma estructural funcionalista.

En 1934 el paradigma sistémico fue recuperado por Ludwig von Bertalanffy (1901-1972), biólogo y filósofo austriaco, bajo la designación de teoría general de los sistemas, título posiblemente inspirado en el libro Foundations of the theory of signs (Fundamentos de una teoría de los signos, 1938), del ingeniero Charles William Morris (1901-1979), así como la influencia del polaco Alfred Korzybski, quien introdujo la teoría de la semántica general. ${ }^{5}$

5 La teoría de la semántica general sostiene que el conocimiento de los seres humanos está limitado por el sistema nervioso central y por la estructura de la lengua. La experiencia del mundo no es directa, depende del sistema nervioso y de la lengua. Lance Strate fue director del Institute of General Semantics y es coautor, con Corey (2012), de un interesante texto sobre Korzybski: Korzybski And... 
Las propuestas de Shannon, Weaver y Bertalanffy fueron determinantes para proyectar el paradigma sistémico hasta afirmarlo como obligado modelo de referencia de proyectos interdisciplinarios, que desbordaron el dominio de las ciencias de la naturaleza y de la vida, comprendiendo al conjunto de las humanidades y de las ciencias sociales. La teoría general de sistemas

\begin{abstract}
afirma que las propiedades de los sistemas no pueden describirse significativamente en términos de sus elementos separados. La compresión de los sistemas sólo ocurre cuando se estudian globalmente, involucrando todas las interdependencias de sus partes. Las tres premisas básicas son las siguientes: Ios sistemas existen dentro de sistemas, los sistemas son abiertos y las funciones de un sistema dependen de su estructura (Wikipedia, s. f. c).
\end{abstract}

En la teoría general de sistemas, resulta fundamental la elaboración de herramientas que permitan capacitar a otras ramas de la ciencia en sus investigaciones prácticas. De ninguna manera la teoría general de sistemas descalifica las ciencias particulares. Fundamentalmente afirma la necesidad de trascender los conocimientos específicos para acceder a una perspectiva amplia e integral, como la realidad. La teoría general de sistemas, además, comprende una metodología específica de la acción global, que completa y corrige los modelos y las metodologías de la acción local.

En la década de 1970, el paradigma sistémico fue recuperado por Niklas Luhmann (1927-1988), quien comprendió la necesidad de extender el alcance explicativo de la teoría general de sistemas al análisis de las sociedades, lo cual lo llevó a polemizar con Jürgen Habermas, uno de los últimos herederos intelectuales de la Escuela de Frankfurt. Los sistemas sociales — sostenía Luhmann — tienden a la desorganización, la cual depende del grado de entropía existente. Los sistemas sociales se mantienen en función de los cambios que establecen con el ambiente, del cual reciben la información y la energía indispensable para mantenerse organizados. Los seres vivos obtienen del ambiente los alimentos que asimilan, y los convierten en energía, que los mantiene vivos. De manera similar, los sistemas sociales retiran del ambiente información que asimilan, y la convierten en un elemento fundamental para su desarrollo. La información les permite organizarse. 
La teoría de Luhmann reposa en el concepto de 'comunicación'. De acuerdo con Luhmann, las unidades constituyentes y reproductoras de los sistemas sociales son las comunicaciones, no los individuos. La sociedad es un tipo de sistema social que comprende todas las comunicaciones: "La comunicación no es una acción humana, ni un fenómeno tecnológico, ni un intercambio de información. Los hombres no pueden comunicar, "solo la comunicación comunica” (Wikipedia, s. f. d). Con Luhmann la teoría general de sistemas afirma sus pretensiones de universalidad, afirmando sus posibilidades de aplicación en todo fenómeno social, como atinadamente destaca Arriaga (2003).

La teoría luhmanniana presenta, en cambio, un poderoso instrumental analítico que permite comprender el funcionamiento de la sociedad, los subsistemas y las organizaciones. Los mecanismos de reducción de la complejidad, los códigos propios de los diferentes subsistemas, los esquemas binarios de selección y regulación de las relaciones al interior de cada subsistema y los intercambios entre ellos, son escudriñados rigurosamente, de tal manera que el aparato conceptual se perfila como una visión muy adecuada a las características de la sociedad moderna y a los procesos que se suceden en sus diferentes niveles.

La complejidad no representa dificultad alguna para un sistema. Al contrario, es una condición indispensable para su desarrollo. Los sistemas surgen del proceso de reducción de complejidad. Los sistemas son menos complejos que sus entornos y sus límites no son físicos, sino de sentido. Para Luhmman, la función antecede a la estructura. Luhmann definía su postura como funcional, estructuralismo, distinguiéndose así del estructural funcionalismo de Talcott Parsons (1902-1979).

Luhmann sostenía que el sistema social utiliza el lenguaje como membrana para tomar del ambiente la información necesaria a fin de codificarla adecuadamente. Lance Strate, quien en la actualidad admite ser considerado como uno de los pilares teóricos de la ecología de los medios, comprendió la estrecha relación que admiten algunas tesis de McLuhan y Luhmann, así como la congruencia que es posible afirmar entre la ecología de los medios, la teoría general de sistemas y la semántica general: 
McLuhan (2003) argumentó que el lenguaje es una forma de percepción, es más, que los idiomas son órganos de la percepción. Y para Luhmann $(1982,1989,1995,2000)$, tanto la percepción como el lenguaje contribuyen al mantenimiento y al funcionamiento de los límites de los sistemas sociales de auto organización (Strate, 2010, p. 35).

Sobre la perspectiva sistémica de Marshall McLuhan, Lance Strate sostiene que la metáfora "galaxia", que Marshall McLuhan empleó en el título de su tercer libro: The Gutenberg Galaxy: The Making of Typographic Man (La Galaxia de Gutenberg: la génesis del hombre tipográfico), admite ser considerada como sinónimo de sistema (Strate, 2004, p. 6). Lance atinadamente señala que el libro Laws of media: the new science ${ }^{6}$ (Leyes de los medios: la nueva ciencia, 1990) afirma plenamente la perspectiva sistémica de la ecología de los medios como de Marshall McLuhan. En el mencionado libro, los McLuhan introdujeron su tétrada, que admite ser considerada como la perfecta conclusión del fecundo trabajo intelectual de Marshall McLuhan. La "tétrada de McLuhan" comprende cuatro leyes que admiten ser aplicadas, sin excepción, a todas las creaciones del hombre, tangibles como intangibles, abstractas o concretas. Las cuatro leyes parten de los siguientes cuestionamientos sobre el impacto de los media y medium en la ecología cultural de las sociedades: ¿qué extienden los medios o tecnologías? ¿Qué vuelven obsoleto? ¿Qué recuperan? ¿Qué revierten? La tétrada sintetiza la perspectiva sistémica y el pensamiento complejo de McLuhan. La representación gráfica de los citados interrogantes es conocida como tétrada (figura 1).

La primera ley, relativa a la extensión, indica que toda tecnología extiende una facultad física o psíquica del hombre. Debemos tener presente que el concepto de 'extensión' supone la posibilidad de prolongar, reemplazar, incrementar, reforzar, acelerar, intensificar. La segunda ley, que corresponde a la obsolescencia o inversión, y es consecuencia directa de la extensión, implica que, cuando un medio prolonga una facultad física o psíquica, determinadas partes del entorno de lo extendido se vuelven obsoletas. Según McLuhan, las tecnologías extienden pero también amputan. En los cambios tecnológicos, las amputaciones resultan inevitables. Mientras

6 El libro fue publicado por Eric - el mayor de los seis hijos que Marshall procreó con Corinne-, en 1988. Eric concluyó el libro, pues Marshall falleció el 31 de diciembre de 1980. De acuerdo con Eric McLuhan, este libro admite ser considerado como la obligada continuación del cuarto libro de McLuhan: Understanding Media: The Extensions of Man. 
determinados sentidos o facultades se incrementan, otros inevitablemente disminuyen. El concepto medular de la tercera ley es la recuperación, que supone entumecimiento. La estructura social resiente los efectos de toda nueva tecnología y paulatinamente recupera su equilibrio. En ocasiones, la recuperación es resultado de adaptaciones o procesos compensatorios. La cuarta ley se define a partir de la reversión. Cuando la tecnología llega a su límite — que en términos mcluhianos significa el sobrecalentamiento del medio-, suelen presentarse hechos y situaciones que revierten o "enfrían” la tendencia. Un medio solo revierte porque se ha llevado al límite.

\section{Figura 1. Tétrada de McLuhan \\ OBJETO DEL ANÁLISIS}

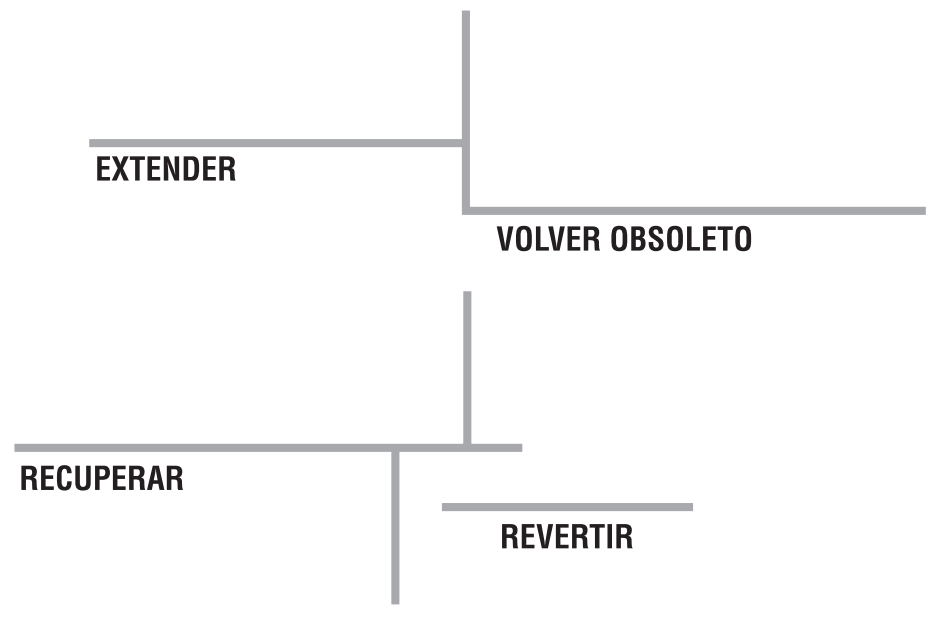

Fuente: Federman y De Kerckhove (2003, p. 103).

\section{Conclusión}

La ecología de los medios, al igual que la teoría general de sistemas, admite ser considerada como una metadisciplina (Gaston, 1986) compleja y sistémica, que, para estudiar los efectos de las tecnologías en la cultura de las sociedades a lo largo de la historia, se ve en la necesidad de involucrar a un amplio número de ciencias, como la gramática, la retórica, la semiótica, la teoría de sistemas, la historia, la filosofía, la cibernética, las ciencias de la comunicación, las artes - la literatura-y, por supuesto, la tecnología misma. La historia de las ciencias, como también la filosofía, representan 
en sí mismas metadisciplinas, afirmó atinadamente Gilles Gaston Granger. El medioecologista es un historiador, un antropólogo, un hombre de letras, un estudioso de la semántica general y un filósofo que analiza los cambios que producen las tecnologías en las sociedades. El objeto de estudio de la ecología de los medios supone un abordaje complejo, simultáneamente semántico, ecológico e histórico.

\section{Referencias}

Arriaga Álvarez, E. G. (2003). La teoría de Niklas Luhmann. Convergencia, 32. Recuperado el 15 de enero de 2015 de http://www.infoamerica.org/documentos_pdf/luhmann_01.pdf

Bacon, F. (2004). Novum organum. Madrid: Losada.

Benito, A. (1982). Fundamentos de la teoría general de la información. Madrid: Pirámide.

Bertalanffy, L., von (1976). Teoría general de los distemas. México: Fondo de Cultura Económica.

Bolter, J. y Grusin, R. (1999). Remediation: Understanding new media. Cambridge, MA: MIT Press.

Carey, J. W. (1989). Communication as culture: Essays on media and society. Boston: Unwin Hyman.

Casey, M. (2000). The intellectual roots of media ecology. The New Jersey Journal of Communication, 8(1), 1-8.

Corey A. y Strate, L. (2012). Korzybski And... Nueva York: Institute of General Semantics.

Crosby, H. H. y Bond, G. R. (1968). The McLuhan explosion: a casebook on Marshall McLuhan and understanding media. Nueva York: American Book Company. 
Coupland, D. (2009). Extraordinary Canadians: Marshall McLuhan. Penguin.

Czitrom, D. (1985). De morse a McLuhan. México: Publigraphics.

Day, B. (1967). The message of Marshall Mcluhan. Londres: Lintas.

De Kerckhove, D. (1997). Inteligencias en conexión: hacia una sociedad de la web. Barcelona: Gedisa.

De Kerckhove, D. (1999). La piel de la cultura: investigando la nueva realidad electrónica. Barcelona: Gedisa.

DeMott, B. (1967). Against McLuhan. En G. Stearn (ed.), Hot and cool a critical symposium. Nueva York: The Dial Press.

Descartes, R. (2011). El discurso del método. Madrid: Alianza Editorial.

Duffy, D. (1969). Marshall McLuhan. Toronto: McClelland and Stewart.

Ellul, J. (1964). The technological society. Nueva York: Vintage Books.

Federman, M. y De Kerckhove, D. (2003). McLuhan for managers. Canadá: Viking.

Fernández, C. y Hernández, R. (2004). Marshall McLuhan, de la torre de control a la torre de marfil. México: Instituto Politécnico Nacional. Recuperado el 15 de enero de 2015 de http:/ /www.razonypalabra.org.mx/ MarshallMcLuhan_DeLaTorreDeMarfilALaTorreDeControl.PDF

Finkelstein, W. (1968). Sense and nonsense of McLuhan. Nueva York: International Publishers.

Fitzgerald,J.(2001).MarshallMcLuhan: wize guy. Montreal:XYZPublishing.

Gaston Granger, G. (1986). ¿Qué es una metadisciplina? Diánoia, 32(32), 103-117. 
Goody, J. (1977). The domestication of the savage mind. Cambridge, Reino Unido: Cambridge University Press.

Gordon, T. (ed.) (2003). Marshall McLuhan Understanding media. The extensions of man. Critical edition. Berkeley, CA: Ginko Press.

Gordon, W. T. (1988). Marshall McLuhan. Escape into understanding: The authorized biography. USA: Basic Books.

Gordon, W. T. (1997). McLuhan for beginners. Nueva York: Writers and readers.

Gordon, W. T. (2003). Understanding media: The extension of man. Critical edition. Germany: Ginko Press.

Gordon, W. T. (2010). McLuhan. USA: Continuum.

Gordon, W. T. (2010). McLuhan: a guide for the perplexed. USA: Continuum.

Gordon, W. T. y Willmarth, S. (1988). McLuhan para principiantes. Buenos Aires: Era Naciente.

Harrocks, C. (2004). McLuhan y la realidad virtual. Barcelona: Gedisa.

Hobbes, T. (2003). Leviatán. Buenos Aires: Losada.

Innis, H. (1952). Changing concepts of time. Toronto: University of Toronto Press.

Innis, H. (1999). The bias of communication. Toronto: University of Toronto Press,

Innis, H. (2007). Empire and communications. Toronto: Dundurn Press.

Islas, O. (2014). 50 años de "Comprender los medios de comunicación. Las extensiones del hombre”, de Marshall McLuhan. Revista Latina 
de Comunicación Social, 69. Recuperado EL 20 de febrero de 2015 de http://www.revistalatinacs.org/069/paper/octavio-islas.html

Kong, C. (2006). Perspectives on culture, technology and communication. USA: Hampton Press.

Korzybski, A. (1994). Science and sanity: An introduction to non-aristotelian systems and general semantics. Nueva York: Institute of General Semantics.

Kroker, A. (1985). Technology and the canadian mind: Innis/McLuhan/Grant. Montreal: New World Perspectives.

Logan, R. (2004). The alphabet effect: A media ecology understanding of the making of western civilization. Nueva York: Hampton Press.

Logan, R. (2010). Understanding new media: Extending Marshall McLuhan. Nueva York: Peter Lang.

Logan, R. (2013). McLuhan misunderstood: Setting the record straight. Toronto: Key Publishing House.

Luhmann, N. (1998). Complejidad y modernidad. Madrid: Trotta.

Luhmann, N. (2006). La sociedad de la sociedad. México: Herder.

Marchand, P. (1989). Marshall McLuhan: The medium and the messanger. Toronto: Random House.

Marx, C. y Engels, F. (2001). Manifiesto comunista. México: Alianza Editorial.

Mattelart, A. (2002). Una historia de la sociedad de la información. Barcelona: Paidós.

McLuhan, E. y McLuhan, M. (2011). Theories of communication. Nueva York: Peter Lang. 
McLuhan, M. (1962). The Gutenberg Galaxy: The making of typographic man. Toronto: University of Toronto Press.

McLuhan, M. (1969). Counterblast. Toronto: McClelland and Stewart.

McLuhan, M. (1970). Culture is our Business. Nueva York: McGraw-Hill.

McLuhan, M. (1996). Comprender los medios de comunicación: las extensiones del ser humano. Barcelona: Paidós.

McLuhan, M. (1999). The medium and the light: Reflections on religion. Toronto: Stodart.

McLuhan, M. (2002). The mechanichal bridge: Folcklore of the industrial man. Nueva York: Vanguard Press.

McLuhan, M. (2005). Marshall McLuhan unbound. Berkeley, CA: Gingko Press.

McLuhan, M. y Carpenter, E. (1970). Explorations in communications. Londres: Cape.

McLuhan, M. y Carson, D. (2003). The book of probes. Nueva York: Ginko Press.

McLuhan, M. y Fiore, Q. (1967). The medium is the message: An inventory of effects. Nueva York: Bantam Books.

McLuhan, M. y Fiore, Q. (1968). War and peace in the global village. Nueva York: Bantam Books.

McLuhan, M. y McLuhan, E. (1988). Laws of media: The new science. Toronto: University of Toronto Press.

McLuhan, M. y McLuhan, E. (2011). Media and formal cause. Texas: NeoPoiesis Press. 
McLuhan, M., McLuhan, E. y Hutchcon, K. (1977). The city as classroom. Ontario: Book Society.

McLuhan, M. y Nevitt, B. (1972). Take today: the executive as dropout. Nueva York: Harcourt Brace.

McLuhan, M. y Parker, H. (1968). Through the vanishing point: Space in poetry and painting. Nueva York: Harper.

McLuhan, M. y Powers, B. (1989). The global village, transformations in world life and media in the 21st century. Nueva York: Oxford University Press.

McLuhan, M. y Watson, W. (2011). From cliche to archetype. Berkeley, CA: Ginko Press.

McLuhan, S. y Staines, D. (eds.). (2003). Marshall McLuhan. Understanding me. Lectures and interviews. Toronto: M\&S.

Meyrowitz, J. (1985). No sense of place: The impact of electronic media on social behavior. Nueva York: Oxford University Press.

Miller, J. (1973). McLuhan. Barcelona: Grijalbo.

Molinaro, M., McLuhan, C. y Toye, W. (eds.) (1987). Letters of Marshall McLuhan. Toronto: Oxford University Press.

Morris, C. (1985). Fundamentos de la teoría de los signos. Barcelona: Paidós.

Morrison, J. C. (2000). Hypermedia and synesthesia. Proceedings of the Media Ecology Association, 1, 37.

Mumford, L. (1961). La ciudad en la historia: sus orígenes, transformaciones y perspectivas. Buenos Aires: Infinito.

Newton, I. (1999). The Principia. Berkeley, California: University of California. 
Pavlik, J. (1998). New media technology: Cultural and commercial perspectives. MA: Allyn \& Bacon.

Postman, N. (1986). Amusing ourselves to death: Public discourse in the age of show business. Nueva York: Penguin.

Postman, N. (s.f.). What is media ecology? En Media Ecology Association. Recuperado el 20 de febrero de 2015 de http:/ /www.media-ecology.org/media_ecology/\#What\%20is\%20Media\%20Ecology?\%20 \%28Neil\%20Postman\%29

Postan, N. (1992). Technopoly: The surrender of culture to technology. Nueva York: Knopf.

Postman, N. y Weingartner, C. (1969). Teaching as a subversive activity. Nueva York: Delacorte.

Rosenthal, R. (ed.). (1968). McLuhan: Pro and Con. Nueva York: Funk \& Wagnalls Co., Inc.

Rushkoff, D. (2006). Screenagers: Lessons in chaos from digital kids. Nueva York: Hampton Press.

Sanderson, F. y Mcdonald, F. (1989). Marshall McLuhan: The man and his message. Colorado: Fulcrum.

Sanderson, G. y McDonald, F. (1989). The unknown McLuhan. Colorado: Fulcrum.

Scolari, C. (2008). Hipermediaciones: elementos para hacer una teoría de la comunicación digital interactiva. Barcelona: Gedisa.

Scolari, C. (2010). Ecología de los medios: mapa de un nicho teórico. Quaderns del CAC, 34(13), 17-25.

Scolari, C. (coord.) (2015). Ecología de los medios: entornos, evoluciones e interpretaciones. Barcelona: Gedisa. 
Shannon, C. (1948). A mathematical theory of communication. Bell System Technical Journal, 27, 379-423 y 623-656.

Shannon, C. (1949). Communication theory of secrecy systems. Bell System Technical Journal, 28, 656-715.

Steam, G. (1967). McLuhan: hot \& cool. Nueva York: The Dial Press.

Strate, L., Jacobson, R. y Gibson, S. (eds.) (2003). Communication and cyberspace: social interaction in an electronic environment. USA: Hampton Press.

Strate, L. (2004). A media ecology review. Communication Research Trends, 23(2), 3-48.

Strate, L. (2010). Korzybski, Luhmann, and McLuhan. Proceedings of the Media Ecology Association, 11, 31-42.

Strate, L. (2014). Amazing ourselves to death: Neil Postman's brave new world revisted. Nueva York: Peter Land.

Strate, L. (s. f.). Media Ecology 101-An Introductory Reading List. Recuperado el 5 de marzo de 2015 de http://www.media-ecology. org/media_ecology/readinglist.html

Surowiecki, J. (2004). The wisdom of crowds. Nueva York: Anchor Books.

Theall, D. (1971). Understanding McLuhan: The medium is the rear view mirror. Montreal: McGill-Queen's University Press.

Theall, D. (2001). The virtual Marshall McLuhan. Montreal: McGill-Queen's University Press.

Toffler, A. (1981). La tercera ola. México: Edivisión. 
Turkle, S. (2011). Alone together: Why we expect more from technology and less from each other. Nueva York: Basic Books.

Wiener, N. (1981). Cibernética y sociedad. México: Consejo Nacional de Ciencia y Tecnología.

Wikipedia (s. f. a). Lance Strate. Recuperado el 5 de marzo de 2015 de https://en.wikipedia.org/wiki/Lance_Strate

Wikipedia (s. f. b). Media ecology. Recuperado 5 de marzo de 2015 de https://en.wikipedia.org/wiki/Media_ecology

Wikipedia (s. f. c). Ludwig von Bertalanffy. Recuperado el 5 de marzo de 2015 de https://es.wikipedia.org/wiki/Ludwig_von_Bertalanffy

Wikipedia (s. f. d). Niklas Luhmann. Recuperado el 5 de marzo de 2015 de https://es.wikipedia.org/wiki/Niklas_Luhmann 
